\title{
Application of SketchUp in Coke Oven Three- Dimensional Digital Modeling
}

\author{
http://dx.doi.org/10.3991/ijoe.v9i2.2581 \\ Shuguang Ouyang, Jie Xu, Xiaoli Su and Zikan Wang \\ Wuhan University of Science and Technology, Wuhan, China
}

\begin{abstract}
Coke oven, which is a large industrial furnace, is complex in structure. A two-dimensional structure diagram can hardly help one observe the inner structure of a coke oven or master its working principle comprehensively. In order to solve this problem, a complete 3D digital model of a coke oven is generated by assembling the three-dimensional models of coke oven components created with SketchUp. It enables users to section the various components of the cove oven. The outer appearance and inner structure of the oven components also can be displayed visually from several different orientations. Moreover, it is convenient to storage and carry, operation easily and fast. It can be displayed on an ordinary computer and occupies no space at the laboratory. Meanwhile, a large sum of money that used for purchasing a physical coke oven model can be saved.
\end{abstract}

Index Terms-SketchUp, Coke Oven, 3D Digital Model, Animation.Introduction

\section{INTRODUCTION}

Coke oven is the main equipment for a coking plant. It is mainly used to produce such chemical products as raw gas and coke which is indispensable for blast furnace ironmaking, cupola melting iron and nonferrous metal smelting by isolating coal from the air for dry distillation $[1,2]$. Coke oven is a large industrial furnace and complex in structure. It is composed of four parts: regenerator, ramps, the coking chamber \& combustion chamber and furnace roof [3]. The structures of all the four parts are complex, especially the ramp area in which thousands of ramps are laid intricately for gas, air and waste gas to pass respectively [4]. There are 56 combustion chambers in a 55 holes JN60 coke oven, each of which has 32 flues. As each flue is connected with two independent ramps, the whole ramp area has 3584 ramps. In addition, a coke oven with two kinds of heating methods includes another 1792 vertical brick gas roads [5].

Therefore, a two-dimensional structure diagram can hardly help one observe the inner structure of a coke oven and master its working principle comprehensively. In order to solve this problem, a set of 3D digital models of coke oven were created in this program with SketchUp software.

The created 3D digital models of coke oven are based on a WH43K coke oven which is designed by the Design and Research Institute of Wuhan University of Science and Technology. The main structural features are: double flue, exhaust gas recirculation and two kinds of heating methods. The models of each part can be classified into two types: the bricklaying model of the coke oven components and the overall model of the coke oven components.

\section{A BRIEF INTRODUCTION OF SKETCHUP}

SketchUp is a 3D design software program developed by@Last Software Company mainly used for 3D modeling. It also has an outstanding performance in modeling technology, material editor, animation creation and post-processing. The modeling process of SketchUp is so easy and fast compare with 3DMAX and PROE that it is widely used in architecture modeling $[6,7]$. It can be used to create photo-realistic images and excellent animation works $[6,8]$. In addition, it is convenient to generate sectional views and dissection animation that used for demonstrating from any orientation. Therefore, it has been widely applied in architecture, planning, landscape, indoor, and industrial design [9].

\section{3D MODELING OF COKE OvEn AND ANIMATION CREATION BASED ON SKETCHUP}

\section{A. 3D Modeling of Ramp Area of a Coke Oven}

The ramp area is the most complex part in a coke oven whose inner structure is extremely irregular with many curved surfaces and notches. Therefore, it is also the most difficult and important part in 3D modeling. The process of generating a 3D model of a coke oven is exemplified by its ramp area in this paper.

The bricklaying model of coke oven components. The modeling of the ramp area is done layer by layer from the bottom to the top. As the ramp area consists of many brick layers, and the bricks in every layer are quiet different in type and size, a large amount of 3D model monomers should be built in accordance with different brick types during the process of building each brick layer, which is shown by a large number of brick components in SketchUp. The process is as follows.

A. Some rectangle bricks are pulled out for later use according to the size of each brick in the CAD drawing.

B. The 3D model of the whole ramp area are generated strictly in accordance with the contours of the ramps in CAD Bricklaying Figure.

C. The above prepared bricks and models of ramps are precisely positioned according to their relative positions. The real brick types are created by using some SketchUp commands such as Intersect with Model. As there are a large number of expansion joints and sliding slits in the ramp area, it is necessary that a large number of guides should be drawn in SketchUp for precise positioning so as to ensure that each brick type is accurate. 
D. Material, texture and color are given to each of the bricks above, which are precisely assembled according to the order of modeling process. Some important parts such as ramps and tube bricks can also be cut as required.

The bricklaying model and cutaway view of the Ramps are shown in Figure 1.

The overall model of coke oven components. Compared with bricklaying model, modeling of the overall structure of coke oven is easier. First, a rough 3D model of the ramp area is drawn according to the overall size of the ramp area in SketchUp. The 3D model of the ramps is built in accordance to the contour and size of ramps. Last, the ramps are generated by using Intersect with Model command after precise positioning, and the overall 3D model of the ramp area is obtained. In the 3D model of overall structure, expansion joints and sliding joint are not presented.

\section{B. 3D Modeling of Other Parts of the Coke Oven}

The other parts of a coke oven such as regenerator, the coking chamber \& combustion chamber and furnace roof

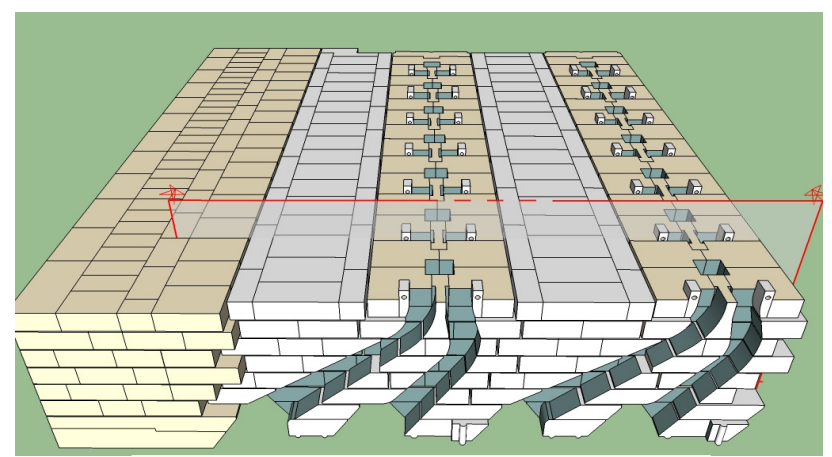

(a) The view of the Ramps.

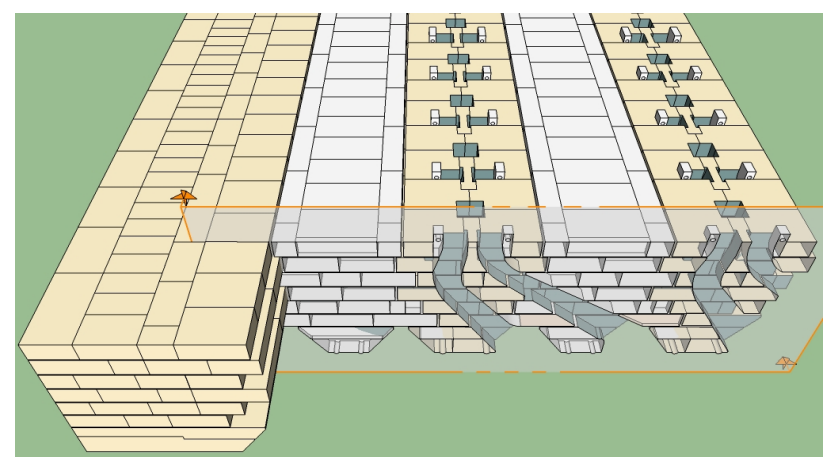

(b) The cutaway view of the Ramps.

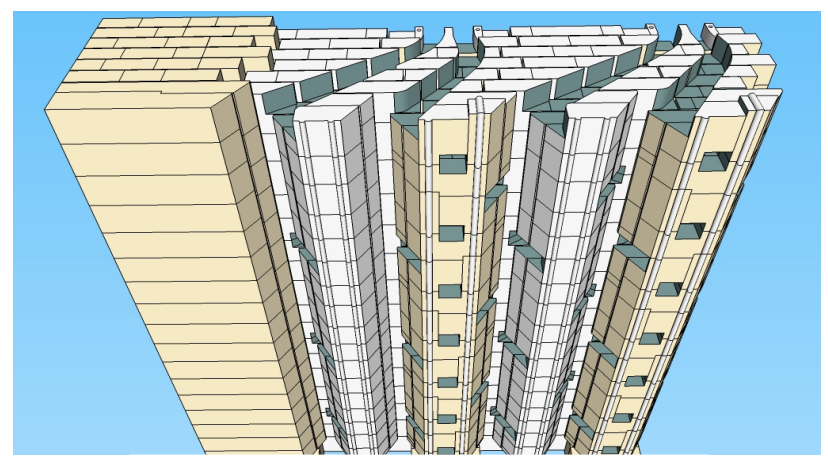

(c) The bottom view of the ramps.

Figure 1. The bricklaying model of the Ramps are simpler in structure when compared with ramp area because they do not have irregular curved surfaces. Consequently, modeling of these parts are much easier, and the needed models can be created only by some basic commands in SketchUp such as Pull/Push, Intersect With Model. The modeling process is similar to the ramp area, which is not repeated here as to the paper length. Models of the various parts of the coke oven are shown in Figures $2,3,4$, and 5 .

\section{Animation CREAtion Based on 3D Digital MODEL OF COKE OVEN}

SketchUp, which owns the feature of animation, can automatically generate smooth animation according to a variety of perspective views which can be set after the scene is created. Its animation is easy to achieve in which the obscure concept of keyframe is avoided [8]. In addition, SketchUp can also conveniently create sectional views from any orientation and generate dissection animation for display [10]. Moreover, the animation can be exported in AVI, Quicktime and MOV files [9].

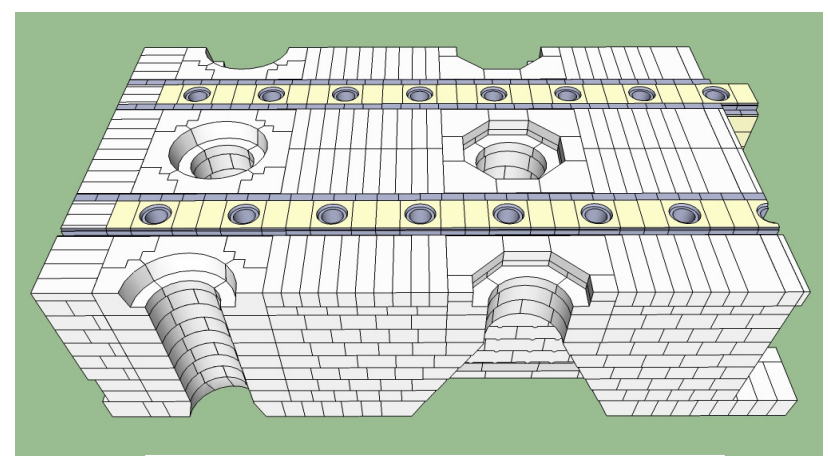

(a) The view of the furnace roof.

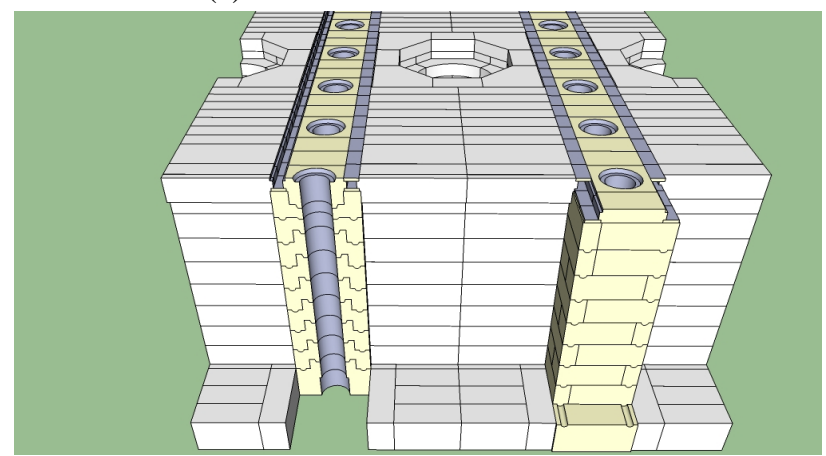

(b) The fire hole of the furnace roof.

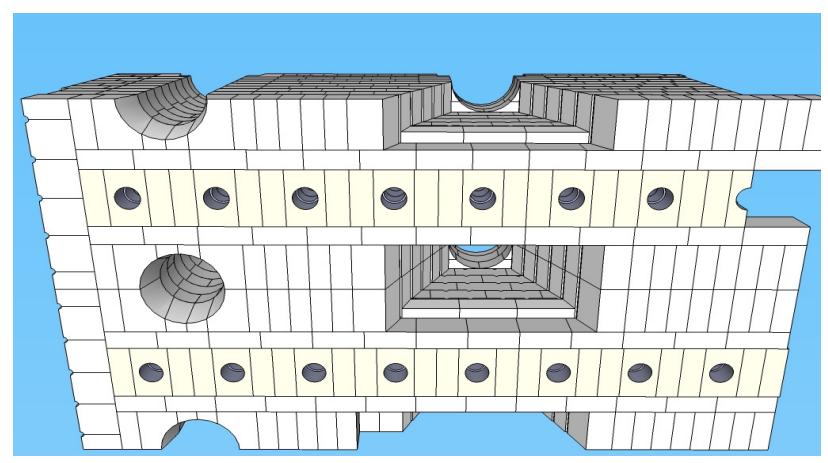

(c) The bottom view of the furnace roof.

Figure 2. The bricklaying model of the furnace roof. 


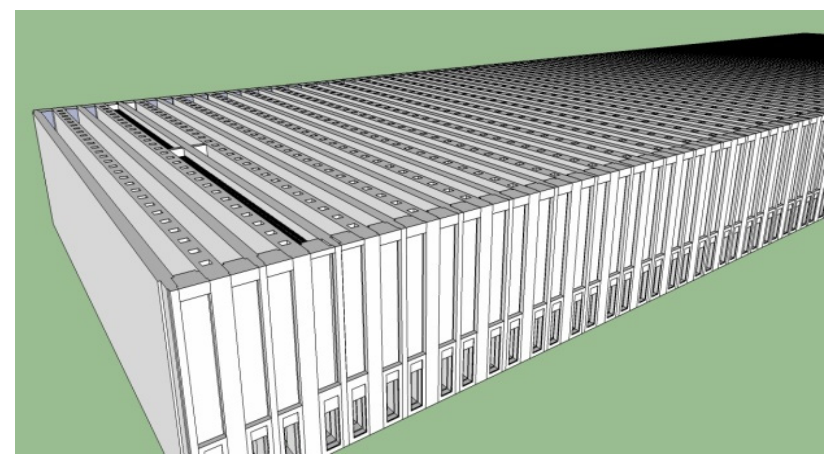

(a) The regenerative chamber's overall model.

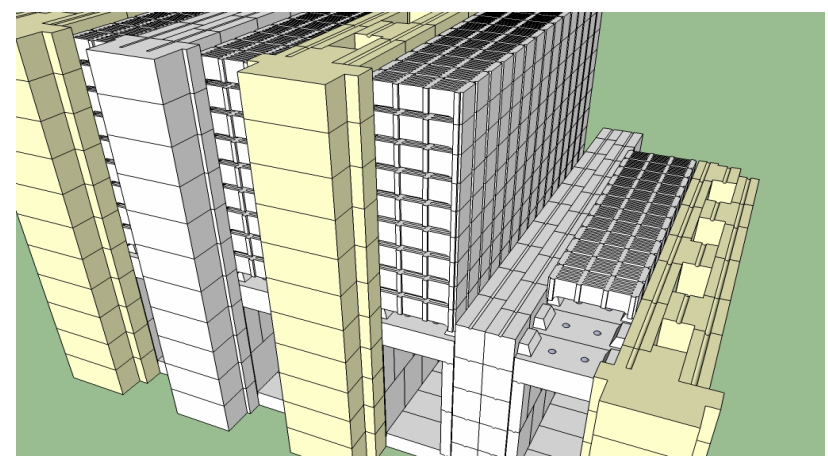

(b) Partial bricklaying model of the regenerative chamber.

Figure 3. The 3D model of the regenerative chamber.

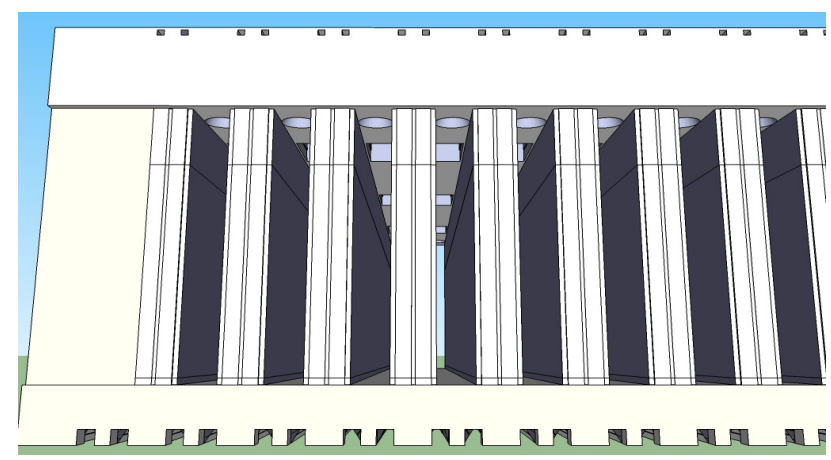

(a) The coking chamber \& combustion chamber's overall model.

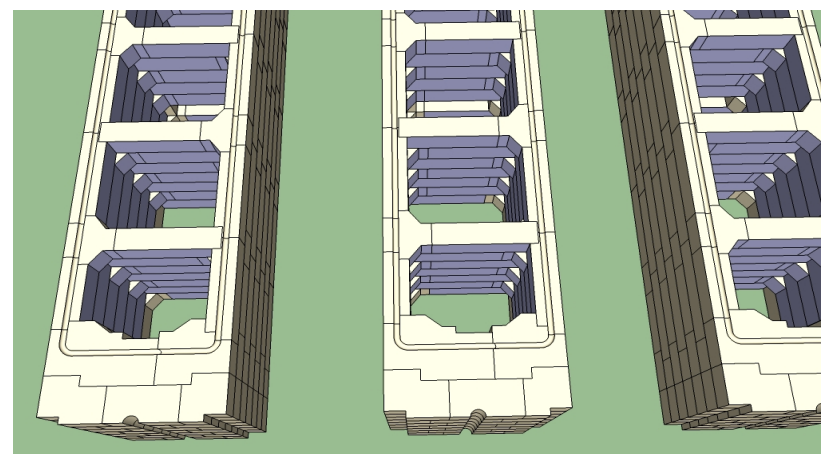

(b) Partial bricklaying model of the coking chamber \& combustion chamber.

Figure 4. The 3D model of the coking chamber\&combustion chamber.

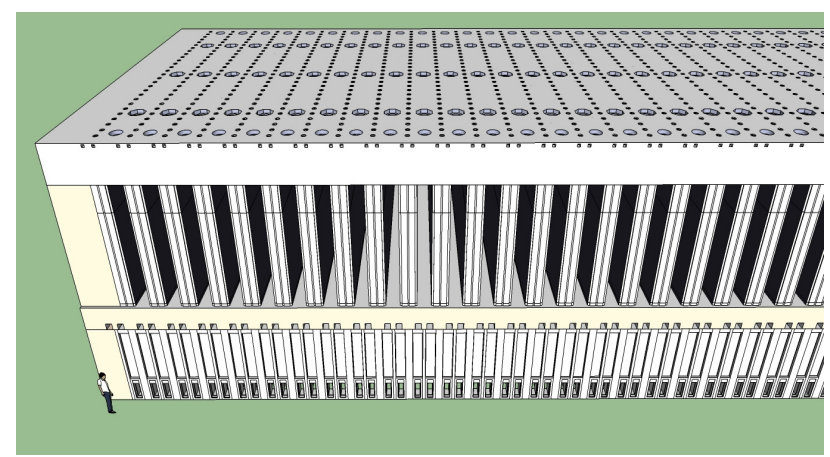

(a) The regenerative chamber's overall model.

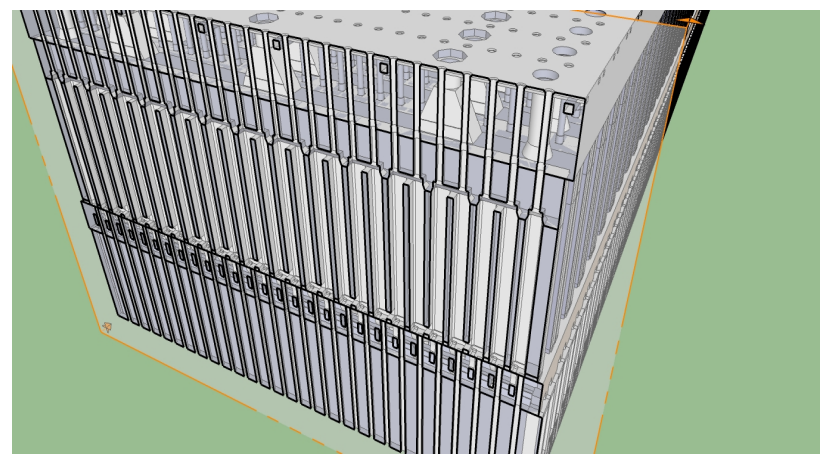

(b) The cutaway view of the coke oven.

Figure 5. The 3D model of the whole coke oven.

In this program, there are $48 \mathrm{AVI}$ animations in total. The content of the animations involve the movement and rotation animation of various parts of the coke oven, dissecting display, the growth animation of the coke oven, as well as the display of the structures of such coke oven parts as checker bricks, grate brick and furnace column etc. The animation vividly displays the relative positions of various parts of the coke oven as well as its complex inner structure.

\section{CONCLUSIONS}

The created 3D digital model has many merits. First, the various parts of the coke oven could be sectioned and visually displayed. It enables users to observe the outer appearance and inner structure of the coke oven from many different perspectives. Second, it is convenient to storage and carry, and it can be displayed on any ordinary computer. Last but not the least, it occupies no space in the laboratory and saves the money that used for purchasing a physical coke oven model.

\section{REFERENCES}

[1] Zhaozhang Yao and Mingdon Zheng, Coking Textbook, 3rd edn., Beijing, CHINA: Metallurgical Industry Press, 2008.

[2] Thomas Henry Byrom and John Edward Christopher, Modern Coking Practice, London: C. Lockwood and son, 1910.

[3] Rainer Worberg and Martin Reinke, "Progress in Coke Oven Technologies", Coke Oven Managers Association, 2009, pp. 1-15.

[4] Zhendon Yu and Wenhua Zheng, Modern coke oven production of technical manuals, Beijing, CHINA: Metallurgical Industry Press, 2010. 
[5] Zhendon $\mathrm{Yu}$ and Chengyou Cai, Coke oven production technology, Shenyang, CHINA: Liaoning Science and Technology Press, 2003.

[6] Daniel Tal, Google SketchUp for site design; a guide to modeling site plans, terrain, and architecture, Hoboken, New Jersey: John Wiley \& Sons, Inc., 2009.

[7] J. Rodríguez, N. Omtzigt, E. Koomen, and F. S. de Blois, "3D Visualisations in Simulations of Future Land Use: Exploring the Possibilities of New, Standard Visualisation Tools", International Journal of Digital Earth, Vol. 1, No. 1, 2008, pp. 148-154. http://dx.doi.org/10.1080/17538940701782718

[8] Tao Wei and Song Wang, SketchUp architectural design, Beijing, CHINA: China Electric Power Press, 2007.

[9] Chris Grover, Google SketchUp: The Missing Manual, Sebastopol, California: O'Reilly Media / Pogue Press, 2009

[10] Sandeep Singh, Beginning Google SketchUp for 3D Printing, New York: Apress, Inc., 2010. http://dx.doi.org/10.1007/978-14302-3362-6

[11] Wei Li, Deren Sheng, and Wei Li, "Development of Fluid Machinery Multimedia Demonstration System Based on 3DS MAX and Authorware", Power System Engineering, Vol. 22, No. 5, 2006, pp. 50-52.

\section{AUTHORS}

Shuguang Ouyang received the B.Sc. and M.Sc. degrees in Chemical Engineering and Technology from the Wuhan University of Science and Technology (WUST), Wuhan, China, in 1992 and 1995, respectively. He is currently a Professor at the College of Chemical Engineering and Technology, Graduate University of the Wuhan University of Science and Technology, Wuhan. His research interests include Coal Conversion, New Carbon Material, Computer Science \& Technology, and 3D Digital Models of Coke Oven. His current research focuses on Image Recognition, Activated Carbon and 3D Digital Models of Coke Oven. He has published more than 30 research articles in refereed journals.
Pro. Ouyang is a member of the Hubei Coal Conversion and New Carbon Material Key Laboratory and was a Co-Chair of Internal Workshop on Modern Science and Technology 2012.. And he is a recipient of 2009 National Science and Technology Progress Awards of China (the Second Award). He is currently with the College of Chemical Engineering and Technology, Wuhan University of Science and Technology, Wuhan, China.

Jie Xu received the B.S. degree in Chemical Engineering and Technology from the Wuhan University of Science and Technology (WUST), Wuhan, China, in 2010. He is currently working towards the M.S. degree at the College of Chemical Engineering and Technology, WUST. His research interests mainly include Cokemaking and 3D Digital Models of Coke Oven.

Xiaoli Su received the B.S. degree in Chemical Engineering and Technology from the Wuhan University of Science and Technology (WUST), Wuhan, China, in 2011. He is currently working towards the M.S. degree at the College of Chemical Engineering and Technology, WUST. His research interests mainly include Activated Carbon and Coking Fly Ash.

Zikan Wang received the B.S. degree in Chemical Engineering and Technology from the Guangdong Ocean University, Guiyang, China, in 2011. He is currently working towards the M.S. degree at the College of Chemical Engineering and Technology, Wuhan University of Science and Technology (WUST), Wuhan, China. His research interests mainly include Coking Fly Ash and 3D Digital Models of Coke Oven.

This article is an extended and modified version of a paper presented at the International Conference on Mechanical Engineering, Automation and Material Science (MEAMS2012), held 22-23 December 2012, Wuhan, China. Received 16 March 2013. Published as resubmitted by the authors 25 March 2013. 